\title{
Clinical adjustability of radiological tools in patients with surgically resected cT1N0-staged non-small-cell lung cancer from the long-term survival evaluation
}

\author{
Hiroaki Kuroda $^{1}$, Takeo Nakada ${ }^{1}$, Yuko Oya ${ }^{1,2}$, Yusuke Takahashi ${ }^{1}$, Hirokazu Matsusita ${ }^{3}$, Noriaki Sakakura ${ }^{1}$ \\ ${ }^{1}$ Department of Thoracic Surgery, Aichi Cancer Center Hospital, Nagoya, Japan; ${ }^{2}$ Department of Thoracic Oncology, Aichi Cancer Center Hospital, \\ Nagoya, Japan; ${ }^{3}$ Division of Translational Oncoimmunology, Aichi Cancer Center Hospital, Nagoya, Japan \\ Contributions: (I) Conception and design: H Kuroda; (II) Administrative Support: T Nakada, H Matsusita; (III) Provision of study materials or \\ patients: H Kuroda, T Nakada, Y Takahashi, N Sakakura; (IV) Collection and assembly of data: H Kuroda, T Nakada, Y Takahashi, N Sakakura; (V) \\ Data analysis and interpretation: H Kuroda, T Nakada, Y Oya; (VI) Manuscript Writing: All authors; (VII) Final approval of manuscript: All authors. \\ Correspondence to: Hiroaki Kuroda. Department of Thoracic Surgery, Aichi Cancer Center Hospital, 1-1, Kanokoden, Chikusa-ku, Nagoya 464-8681, \\ Japan. Email: h-kuroda@aichi-cc.jp.
}

\begin{abstract}
Background: Various radiological tools have been introduced to determine the malignancy or prognosis of lung carcinomas. We retrospectively summarized the clinical outcomes to evaluate whether radiological tools such as consolidation-to-tumor ratio (CTR), tumor disappearance ratio (TDR), and mediastinal diameter (MD) are suitable for surgically resected non-small-cell lung cancer (NSCLC).

Methods: This retrospective study included 260 patients (128 men and 132 women; median age, 64 years) with cT1N0-staged NSCLC who underwent thoracotomy. Disease-free survival (DFS) and overall survival (OS) outcomes were analyzed using the Kaplan-Meier method and Cox proportional hazards model.

Results: When the adjusted hazard ratios (HRs) with reference to cT1a/1 mi were calculated, significant differences were observed in cT1b and cT1c for DFS ( $\mathrm{P}=0.04$ and $\mathrm{P}<0.01$, respectively) and in cT1c for OS $(\mathrm{P}=0.01)$. For HRs with reference to CTR $(\leq 0.5)$, a significant difference was only observed in CTR $(>0.5)$ for DFS $(\mathrm{P}=0.01)$. For HRs with reference to TDR $(\leq 25 \%)$, significant differences were observed in TDR $(>75 \%)$ for DFS $(\mathrm{P}=0.02)$ and $\mathrm{OS}(\mathrm{P}=0.02)$. For HRs with reference to $\mathrm{MD}(\leq 5 \mathrm{~mm})$, significant differences were observed in 6-20 mm (P=0.04) and $>20 \mathrm{~mm}(\mathrm{P}=0.02)$ for DFS and in $>20 \mathrm{~mm}(\mathrm{P}=0.02)$ for OS.

Conclusions: All radiological tools revealed significant correlations with prognosis in the patients with cT1N0-staged NSCLCs. We recommend the use of MD in a clinical context. However, further investigation of this issue is needed.
\end{abstract}

Keywords: Mediastinal diameter (MD); consolidation; non-small cell lung cancer (NSCLC); tumor disappearance rate (TDR); consolidation-to-tumor ratio (CTR)

Submitted Apr 08, 2020. Accepted for publication Sep 10, 2020.

doi: $10.21037 /$ jtd-20-1610

View this article at: http://dx.doi.org/10.21037/jtd-20-1610

\section{Introduction}

Recently, high-resolution computed tomography (HRCT) has become important for clarifying malignant behavior in clinical practice. Various studies have revealed the correlation between the proportion of ground-grass opacity component and the pathological features of lung adenocarcinoma, such as consolidation-to-tumor ratio and tumor disappearance ratio (TDR), before the 8th edition of the TNM classification (1). Several authors reported that TDR as well as CTR had closely correlations with malignant behavior and prognosis (2,3). A little later after TDR or CTR, direct measurement of solid components such as consolidation diameter (CD) or mediastinal diameter (MD) was closely associated with lymph node 
involvement and unfavorable postsurgical outcomes because the pathological development of the central fibrosis was also confirmed to be an important prognostic factor for lung adenocarcinomas $(4,5)$. However, we previously illustrated that the pathological invasive region was composed of two areas: alveolar thickening showing lepidic growth and nonlepidic/fibroblastic foci (6). Moreover, Saito et al. reported that the histopathological findings of small pulmonary adenocarcinomas could be classified into three groups: collapse with or without bronchioloalveolar cell carcinoma, fibrosis, and mucus type (7).

Currently, "T" category is defined by CD in HRCT; this was included for the first time in the 8th TNM edition (1). It is not easy to determine the solid component using HRCT because of the existence of the background of normal lung. We previously reported that the size of lung adenocarcinoma, evaluated using MD on HRCT, is a more important predictive prognosis factor than the total tumor size or CD (8). In this context, the following question arises: Which method is more appropriate to obtain a proxy indicator of the malignancy and prognosis? From an analysis of 324 adenocarcinomas, we previously postulated that CD as well as MD was superior to pathological node positivity rather than pathological invasive size (8). Recently, Kim et al. reported that CTR and TDR were not independent prognostic factors and concluded that preoperative prognostication based on clinical $\mathrm{T}$ category would be sufficient without further stratification (9). However, this contradicts previous reports from retrospective studies. Therefore, we examined the efficacy of radiological tools (CTR, TDR, and MD) on disease-free survival (DFS), and overall survival (OS) in patients with surgically resected cT1N0-staged non-small cell lung cancer (NSCLC), especially in those with a long follow-up period and thoracotomy. We present the following article in accordance with the STROBE reporting checklist (available at http:// dx.doi.org/10.21037/jtd-20-1610).

\section{Methods}

\section{Patients}

In this study, we retrospectively evaluated 289 patients who underwent curative thoracotomy lobectomy or segmentectomy for clinical stage I NSCLC at a single Japanese institution between March 2006 and March 2010. This study was conducted in accordance with the Declaration of Helsinki (as revised in 2013) and approved by the institutional review board of Aichi Cancer Center, Aichi Prefecture, Japan (2019-1-544). Informed consent was waived due to the retrospective nature of this study. The following exclusion criteria were applied: pure groundglass nodules on HRCT; wedge resection; and pathological proven small cell lung cancer.

We collected patient data as follows: age, sex, smoking history, surgical procedure, oncogene [epidermal growth factor receptor (EGFR), and KRAS], and T category assessed using HRCT. DFS, and OS were defined as the interval from the date of surgery to the date of cancer recurrence, death, or the final follow-up. The patients were followed up every 3 or 4 months for the first 3 years postoperatively and every 4 to 6 months thereafter. For all patients, the presence of potential distant metastasis was assessed preoperatively using chest CT, abdominal CT or ultrasonography, brain CT or magnetic resonance imaging, and either bone scintigraphy or PET. Clinically, the mediastinal and hilar nodal status was deemed positive if the chest CT findings revealed a nodal short axis of at least $1.0 \mathrm{~cm}$. The clinical stage was redefined in accordance with the 8th edition of the TNM Classification of Malignant Tumors (1). Histopathological diagnoses were based on the 2004 classification of the World Health Organization (10).

\section{Evaluation of T category using HRCT}

The CT images were assessed using Synapse Vincent threedimensional analysis system (Fujifilm Medical Co., Ltd., Tokyo, Japan) as a workstation and reviewed by members of our thoracic oncology department in consensus. Two different tumor axial dimensions were evaluated on CT imaging in accordance with our previous reports: (I) CD: lung setting [level, -600 Hounsfield units (HU); width, 1,500 HU] and (II) MD: mediastinal setting (level, $35 \mathrm{HU}$ without enhancement or $75 \mathrm{HU}$ with enhancement; width, $320 \mathrm{HU}$ (6). The CTR and TDR were calculated using the following formulas: CTR: CD/whole-tumor diameter; and TDR (\%): [1 - (MD/whole-tumor diameter) $\times 100$.

\section{Statistical analyses}

All computations were performed using standard SPSS v25.0 software (SPSS Inc., IBM, Chicago, IL, USA). We evaluated the association between Clinical $\mathrm{T}$ category, CTR, TDR, and MD as radiological implements and patient outcomes (DFS and OS). The survival rates of the subgroups of patients were analyzed using the Kaplan- 


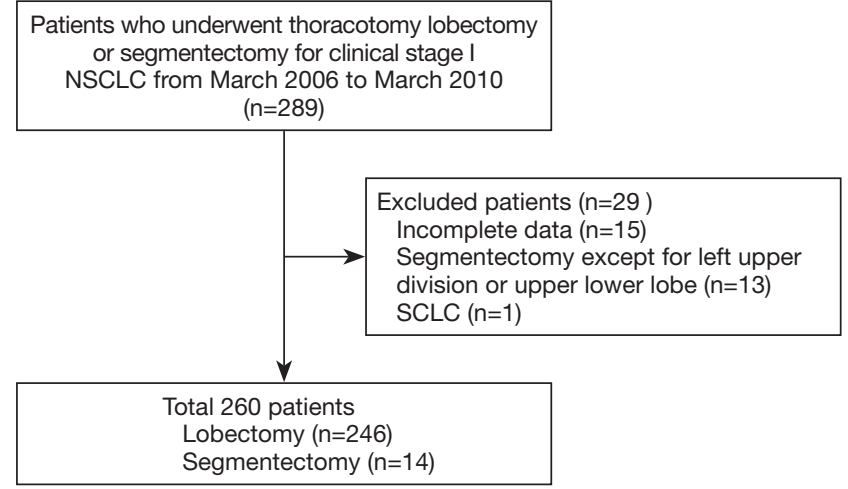

Figure 1 Flow chart for patient selection.

Meier method. The differences between groups were assessed using the log-rank test and Cox's proportional hazards model. A P value $<0.05$ was considered statistically significant.

\section{Results}

\section{Patients characteristics}

A total of the 289 cT1N0-staged NSCLCs were collected and the analyses were finally performed in 260 (90.0\%) patients (Figure 1). Twenty-nine patients were excluded, of which $28(9.7 \%)$ underwent wedge resection, and 1 $(0.3 \%)$ had pathologically proven small cell lung cancer. Their relevant characteristics are summarized in Table 1. In our cohort, 128 (49.2\%) were male and 132 (51.8\%) were female, with a mean age of 64 years. The patients underwent thoracotomy lobectomy in $246(94.6 \%)$ and segmentectomy in $14(5.4 \%)$. The mean follow-up duration after surgery was $83.8 \pm 39.6$ months (range, 4.1-160.0 months). The $\mathrm{cN} 0$-staged NSCLCs were categorized in the following clinical $\mathrm{T}$ categories in Table 1 . The incidences of various radiological implements and mutation status corresponded to clinical T categories in Table 2.

\section{Clinical outcomes (Figures 2,3)}

The 5-year DFS and OS rates for cN0-staged NSCLC after thoracotomy were $81.4 \%$ and $85.8 \%$, respectively. The prognosis stratified by clinical $\mathrm{T}$ categories indicated 5 -year DFS and OS rates of $100 \%$ and $90.0 \%$ for cT1mi, $94.9 \%$ and $94.7 \%$ for $\mathrm{cT} 1 \mathrm{a}, 84.8 \%$ and $89.2 \%$ for $\mathrm{cT} 1 \mathrm{~b}$, and $69.2 \%$ and $77.3 \%$ for cT1c, respectively $(\mathrm{P}=0.015$ and $\mathrm{P}<0.001$ ) (Figures $2 A, 3 A$ ).
Table 1 The patient characteristics

\begin{tabular}{|c|c|}
\hline Variables & Outcome, $\mathrm{n}=260$ \\
\hline Sex, male/female & $128 / 132(49.2 \%)$ \\
\hline Age (years/old, mean, range) & $64 \pm 9[32-84]$ \\
\hline Pack-year (mean) & $16.1 \pm 22.9[0-110]$ \\
\hline \multicolumn{2}{|l|}{ Procedures } \\
\hline Segmentectomy & $14(5.4 \%)$ \\
\hline Lobectomy & $245(94.2 \%)$ \\
\hline Bilobectomy & $1(0.4 \%)$ \\
\hline \multicolumn{2}{|l|}{ Clinical T category } \\
\hline T1mi & $11(4.2 \%)$ \\
\hline T1a & $41(15.8 \%)$ \\
\hline $\mathrm{T} 1 \mathrm{~b}$ & $113(43.5 \%)$ \\
\hline $\mathrm{T} 1 \mathrm{c}$ & 95 (36.5\%) \\
\hline \multicolumn{2}{|l|}{ Consolidation-to-tumor } \\
\hline Mean & $0.75 \pm 0.25$ \\
\hline$\leq 0.5$ & $46(17.7 \%)$ \\
\hline$>0.5$ & $214(82.3 \%)$ \\
\hline \multicolumn{2}{|l|}{ Tumor disappearance ratio (\%) } \\
\hline$\leq 25$ & $103(39.6 \%)$ \\
\hline $26-75$ & $113(43.5 \%)$ \\
\hline$>75$ & $44(16.9 \%)$ \\
\hline \multicolumn{2}{|l|}{ Mediastinal diameter (mm) } \\
\hline Mean & $13.8 \pm 8.1$ \\
\hline$\leq 5$ & $43(16.5 \%)$ \\
\hline $6-20$ & $149(57.3 \%)$ \\
\hline$>20$ & $68(26.2 \%)$ \\
\hline \multicolumn{2}{|l|}{ Mutations } \\
\hline EGFR (yes/no/unknown) & $137 / 115 / 8$ \\
\hline KRAS (yes/no/unknown) & $14 / 237 / 9$ \\
\hline
\end{tabular}

In the CTR subgroup analysis, the 5 -year DFS and OS rates were $95.5 \%$ and $93.0 \%$, and $78.3 \%$ and $84.2 \%$ in the $\leq 0.5(\mathrm{n}=46)$ and $>0.5$ groups $(\mathrm{n}=214)$, respectively (Figure $2 A, B)$. A significant difference was observed in DFS $(\mathrm{P}=0.003)$, but not in OS $(\mathrm{P}=0.052)$ (Figures $2 B, 3 B)$.

In the TDR subgroup analysis, the 5 -year DFS and OS rates were $70.5 \%, 86.0 \%$, and $95.1 \%$, and $79.0 \%, 89.4 \%$, and $92.4 \%$ in the $\leq 25,26-75$, and $>75$ TDR groups, 
Table 2 The distribution of various implements and mutations

\begin{tabular}{|c|c|c|c|c|}
\hline Variable & \multicolumn{4}{|c|}{ CT category } \\
\hline \multicolumn{5}{|c|}{ Consolidation-to-tumor ratio } \\
\hline$\leq 0.5 ; n=46,17.7 \%$ & $11(2.4 \%)$ & $25(5.4 \%)$ & $10(2.2 \%)$ & $0(0 \%)$ \\
\hline$>0.5 ; n=214,82.3 \%$ & $0(0 \%)$ & $16(7.5 \%)$ & $103(48.1 \%)$ & $95(4.4 \%)$ \\
\hline$>75 ; n=44,16.9 \%$ & $10(22.7 \%)$ & $24(54.5 \%)$ & $9(20.5 \%)$ & $1(2.3 \%)$ \\
\hline $26-75 ; n=113,43.5 \%$ & $1(0.9 \%)$ & $16(14.2 \%)$ & $69(61.0 \%)$ & $27(23.9 \%)$ \\
\hline$\leq 25 ; n=103,39.6 \%$ & $0(0 \%)$ & $1(1 \%)$ & $35(34.0 \%)$ & $67(65.0 \%)$ \\
\hline \multicolumn{5}{|c|}{ Mediastinal diameter (mm) } \\
\hline$>20, n=68,26.2 \%$ & $0(0 \%)$ & $0(0 \%)$ & $0(0 \%)$ & $68(100 \%)$ \\
\hline \multicolumn{5}{|l|}{ Mutations } \\
\hline EGFR; $n=137 ; 54.3 \%$ & $9(6.6 \%)$ & $28(20.4 \%)$ & $60(43.8 \%)$ & $40(29.2 \%)$ \\
\hline KRAS; $n=14,5.6 \%$ & $0(0 \%)$ & $3(21.4 \%)$ & $7(50 \%)$ & $4(28.6 \%)$ \\
\hline
\end{tabular}
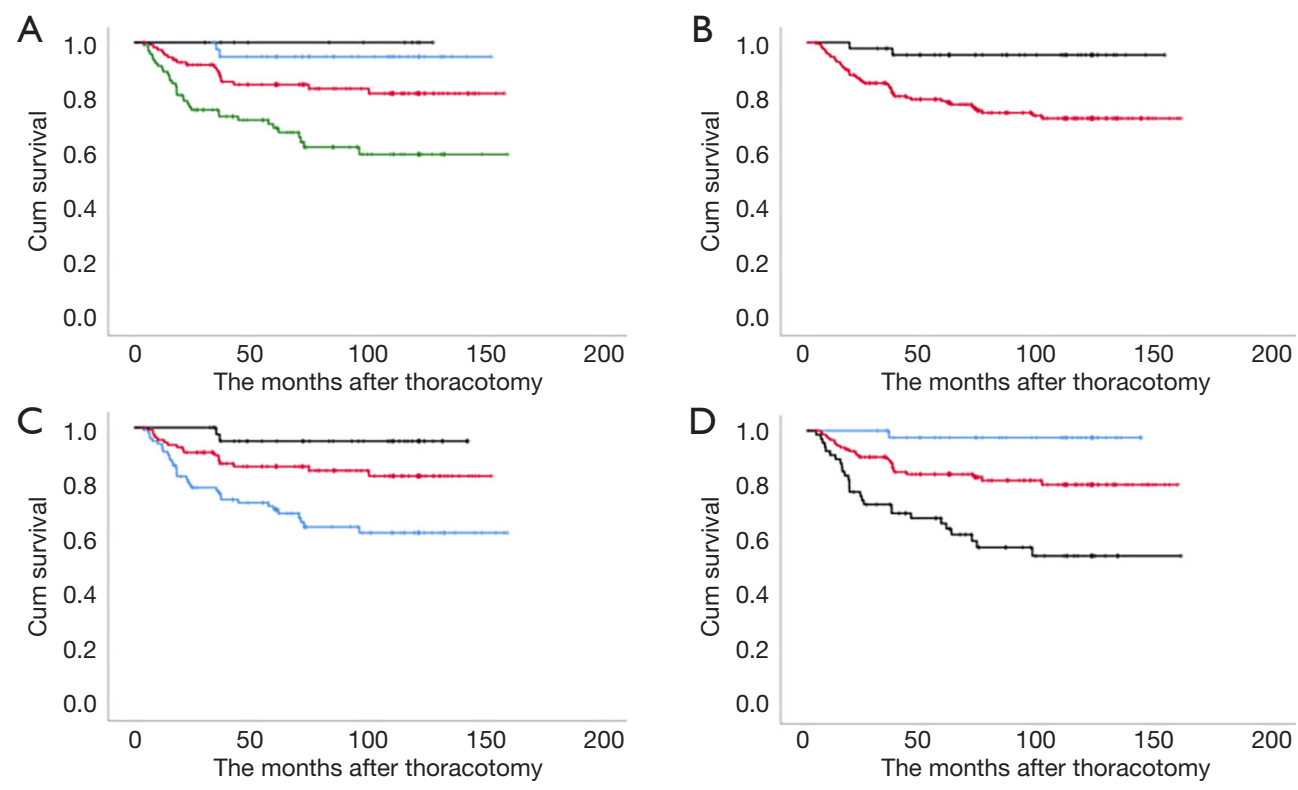

Figure 2 Kaplan-Meier graphs of the disease-free survival in patients with clinical T1N0 staged surgically resected non-small cell lung cancers according to various radiological tools. (A) To clinical 'T' category. Black: T1mi; blue: T1a; red: T1b, and green: T1c. (B) To consolidation-to-tumor ratio. Black: $\leq 0.5$ and red: $>0.5$. (C) To tumor disappearance ratio (\%). Black: $>75$; red: 26-75, and blue: $\leq 25$. (D) To mediastinal diameter (mm). Blue: $\leq 5$; red: 6-20, and black: $>20 . \mathrm{P}<0.05$ indicates significant difference. 

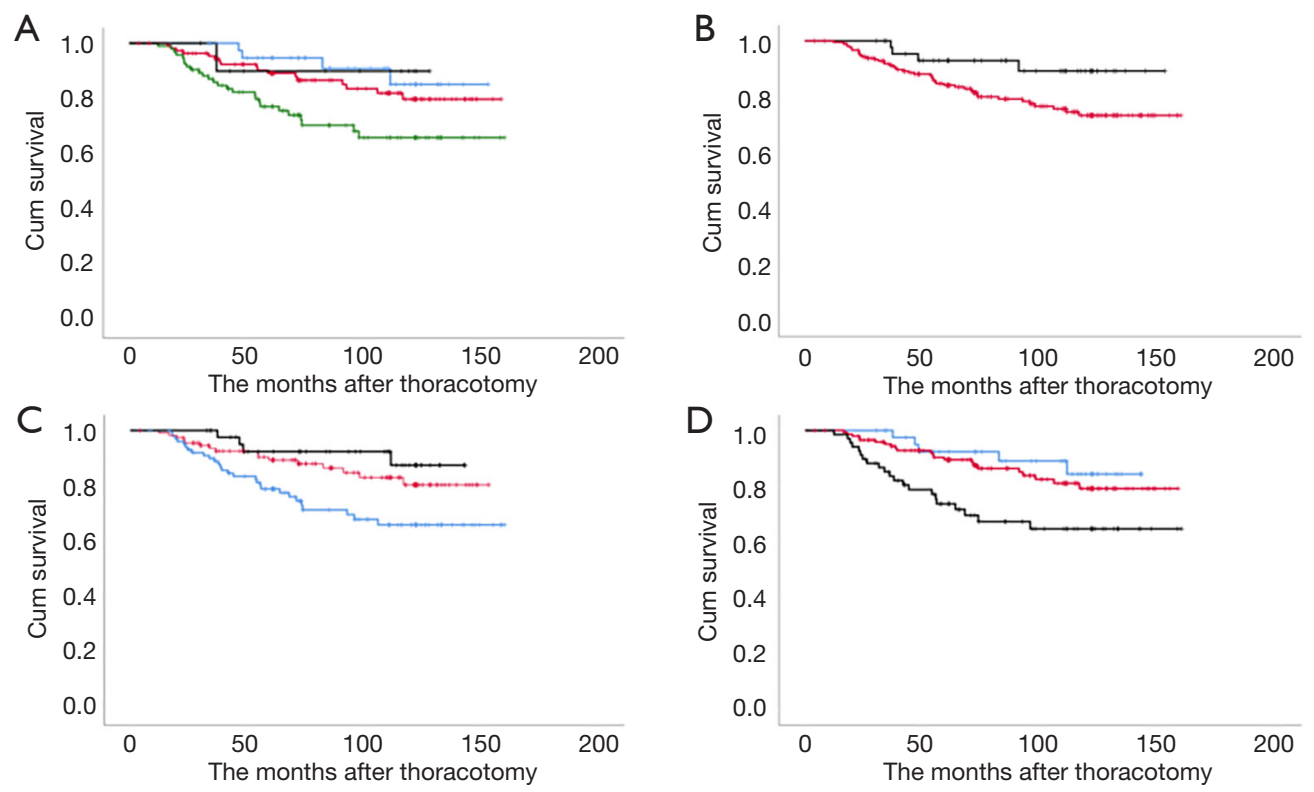

Figure 3 Kaplan-Meier graphs of the overall survival in patients with clinical T1N0 staged surgically resected non-small cell lung cancers according to various radiological tools. (A) To clinical 'T' category. Black: T1mi; blue: T1a; red: T1b, and green: T1c. (B) To consolidationto-tumor ratio. Black: $\leq 0.5$ and red: $>0.5$. (C) To tumor disappearance ratio (\%). Black: $>75$; red: 26-75, and blue: $\leq 25$. (D) To mediastinal diameter (mm). Blue: $\leq 5$; red: 6-20, and black: $>20$. $\mathrm{P}<0.05$ indicates significant difference.

respectively (Figures $2 C, 3 C$ ). Significant differences were observed in both DFS and OS (both $\mathrm{P}<0.01$ ) between the $\leq 75$ and $>75$ groups. Significant differences were also observed in both DFS and OS (both $\mathrm{P}<0.01$ ) between the $\leq 50$ and $>50$ groups.

In the MD subgroup analysis, the 5-year DFS and OS rates were $97.5 \%, 92.4 \%$, and $84.3 \%$, and $89.5 \%, 64.7 \%$, and $73.7 \%$ in the $\leq 5,6-20$, and $>20 \mathrm{~mm} \mathrm{MD}$ groups, respectively (Figure $2 B$ ). Significant differences were observed in both DFS and OS $(\mathrm{P}<0.001$ and $\mathrm{P}=0.005)$ (Figures 2D,3D).

We finally calculated the estimated hazard ratios (HRs) for poor OS according to radiological parameters (Table 3). After adjustment for potential confounding factors, the risk of poor DFS and OS increased gradually for each radiological parameter, with the significantly poorest DFS being associated with $>0.5$ in CTR $(\mathrm{P}=0.01)$, with $\leq 25$ in TDR $(\mathrm{P}=0.02)$, and with $6-20$ and $>20 \mathrm{~mm}$ in $\mathrm{MD}(\mathrm{P}=0.04$ and $\mathrm{P}<0.01)$. Significantly increased risks of poor $\mathrm{OS}$ were observed for $\leq 25$ in TDR $(\mathrm{P}=0.02)$, and $>20 \mathrm{~mm}$ in $\mathrm{MD}$ $(\mathrm{P}=0.02)$, but not for $>0.5$ in CTR $(\mathrm{P}=0.62)$. According to $\mathrm{cT}$ category, significant adjusted $\mathrm{HR}$ with reference to cT1mi/1a was observed in cT1b $(\mathrm{P}=0.04)$ and cT1c $(\mathrm{P}<0.01)$ for DFS and in cT1c $(\mathrm{P}=0.01)$ for OS.

\section{Discussion}

This study was established to investigate the surgical outcomes for cN0-staged non-small-cell lung cancer (NSCLC) after thoracotomy lobectomy and segmentectomy (left upper division, and superior lower lobe). We retrospectively announced our institutional surgical outcomes; adjusted HRs of the 8th TNM classification were 0.63 in pT1a, 1.79 in pT1b, and 1.42 in pT1c, which were compatible with the results of this study (11). However, stage IA1-3 subgroups were not clearly stratified in Sakakura's study, and only 3\% of their patients were classified in IA1 (11). In this study, we also excluded patients who underwent wedge resection segmentectomy except for left upper division, and superior lower lobe, and only $4.3 \%$ of patients were finally eligible as cT1miN0M0. Therefore, we considered that our data could support whether the radiological tools are appropriate for shedding light on malignancy and prognosis.

Among practitioners worldwide, consolidation size on HRCT is believed to be an index predictive of malignant behavior for NSCLCs (1). Many researchers from Japan have reported that CTR and TDR were significantly associated with the surgical outcomes of early-stage NSCLCs via lobectomy or segmentectomy with relatively 
Table 3 Association between the radiological implements and patient outcomes

\begin{tabular}{|c|c|c|c|c|}
\hline Variables & \multicolumn{2}{|c|}{ DFS } & \multicolumn{2}{|c|}{ OS } \\
\hline \multicolumn{5}{|c|}{ Clinical T category } \\
\hline $\mathrm{cT} 1 \mathrm{mi} / 1 \mathrm{a}$ & Reference & - & Reference & - \\
\hline cT1b & $4.96(1.07-19.8)$ & $0.04^{*}$ & $1.68(0.62-4.56)$ & 0.31 \\
\hline \multicolumn{5}{|c|}{ Consolidation-to-tumor ratio } \\
\hline$\leq 0.5$ & Reference & - & Reference & - \\
\hline$>0.5$ & $6.37(1.55-26.2)$ & $0.01^{*}$ & $2.65(0.95-7.38)$ & 0.62 \\
\hline \multicolumn{5}{|c|}{ Tumor disappearance ratio (\%) } \\
\hline$\leq 25^{\#}$ & $9.11(2.19-38.0)$ & $0.02^{*}$ & $3.30(1.24-8.75)$ & $0.02^{*}$ \\
\hline \multicolumn{5}{|c|}{ Mediastinal diameter (mm) } \\
\hline$\leq 5$ & Reference & - & Reference & - \\
\hline $6-20$ & $8.27(1.12-61.1)$ & $0.04^{*}$ & $1.68(0.62-4.58)$ & 0.45 \\
\hline$>20$ & $22.3(3.03-164.2)$ & $0.02^{*}$ & $3.51(1.35-9.15)$ & $0.02^{*}$ \\
\hline
\end{tabular}

*, $\mathrm{P}<0.05$; ", indicates significant difference. HR, hazard ratio; $\mathrm{Cl}$, confidence intervals.

large-scale analyses $(2,3)$. However, in a recent report, it was postulated that CTR and TDR were not independent prognostic factors, and that preoperative prognostication based on clinical $\mathrm{T}$ category would be sufficient without further stratification according to CTR or TDR (9). Their nodule measurements were performed by two trained radiology technicians, who has one, or seven years of research experience in chest CT. In this study, adjusted HRs of the 8th TNM classification were 4.96 in pT1b and 11.6 in pT1c with reference to cT1mi/1a in DFS, and 1.68 in $\mathrm{pT} 1 \mathrm{~b}$ and 3.51 in pT1c with reference to $\mathrm{cT} 1 \mathrm{mi} / 1 \mathrm{a}$ in OS, which was compatible with the results of the study by Kim et al. From February 2016, preoperative evaluation of clinical $\mathrm{T}$ by the consolidation size of the lesion on HRCT was adopted worldwide $(12,13)$. The ground glass tumor size has played a very important role in preoperative clinical evaluation, especially in cT1; this recent recognition would affect the assessment of cT1 lesions with a lepidic component to a greater extent. Finally, the estimated HRs for DFS, and OS were calculated according to Clinical T category, CTR, TDR, and MD as radiological parameters (Table 3), layered parameter using consolidation size and/ or MD could contribute superior prognostic parameter compared to those of CTR or TDR.

Several reports described that CTR and TDR were not prognostic parameters for clinical stage I lung adenocarcinomas $(9,14-16)$. Nowadays, it is considered that the size of the CD itself is important as a prognostic factor in terms of tumor morphology, whereas the CTR and TDR relatively evaluate the component of the consolidation to the overall tumor diameter. For the total adenocarcinomas, clinical $\mathrm{T}$ category was better radiological prognostic tool (9). We previously reported that either consolidation size or MD was superior to pathological invasive size for upstaging due to lymph node metastasis in 324 adenocarcinomas (17). In this study, significant differences were observed in both DFS and OS, especially for $\mathrm{MD}>20 \mathrm{~mm}$ (both $\mathrm{P}=0.02$ ). Additionally, in 2014, we analyzed 176 small adenocarcinomas (10$30 \mathrm{~mm}$ in the maximum diameter) and reported that the 5 -year DFS according to MD subcategories was $98.1 \%, 71.0 \%$, and $49.0 \%$ for $\mathrm{MD}$ of $\leq 10,11-15$, and $>15 \mathrm{~mm}$, respectively (8). Consequently, we investigated the relationship between radiological findings and 
pathological invasive size. In 2016, we analyzed 360 completely resected cT1a-2aN0 adenocarcinomas and proposed that the approximate pathological invasive size was roughly the $\mathrm{MD}+3 \mathrm{~mm}$ (6). Meanwhile, we reported that a pathological invasive size of $\leq 5$ or $>5 \mathrm{~mm}$ was best predicted by MD (AUC $=0.941$ ), followed by CD (AUC $=0.897$ ), with prediction by MD being significantly better than that by $\mathrm{CD}(\mathrm{P}=0.001)$. We also asserted that the recorded CD could vary due to subjectivity in the measurement among physicians, so it cannot always be accurately determined in clinical practice (6). Since the CT Hounsfield number fluctuates according to the pathological subtype because definitive thresholds have not been determined (18), we considered that MD might have a smaller margin of error in terms of its recognition and measurement than $\mathrm{CD}$.

This study had some limitations. First, it involved a retrospective setting based on a single center. However, this work had the strength that the study population was relatively acceptable, and we did not need to rule out inherent potential bias with respect to the surgical indication for thoracoscopy surgery because thoracotomy was performed in all cases. Second, we used the MD evaluation in clinical practice with high affinity, resulting in the avoidance of overestimation and measurement errors that may occur when assessing CD. Third, the patients in this study were not necessarily diagnosed as $\mathrm{cN} 0$ preoperatively using PET evaluation.

In conclusion, all radiological tools significantly revealed correlations with prognosis in patients with cT1N0-staged NSCLCs. Our study suggests that clinical T category might be sufficient as an index of preoperative predictive prognosis in our surgical population. We recommend the MD classification because of its smaller margin of error and that it accurately reflects prognosis. We suggested that NSCLCs with larger MD should be planned more increased surveillance. However, further investigation of this should be performed.

\section{Acknowledgments}

The authors thank Professor Yukinori Sakao, MD, PhD (Department of Thoracic Surgery, Teikyo University, Tokyo, Japan) for the support with the supervision of the manuscript.

Funding: None.

\section{Footnote}

Reporting Checklist: The authors have completed the STROBE reporting checklist. Available at http://dx. doi. org/10. 21037/jtd-20-1610

Data Sharing Statement: Available at http://dx. doi. org/10. 21037/jtd-20-1610

Peer Review File: Available at http://dx. doi. org/10.21037/ jtd-20-1610

Conflicts of Interest: All authors have completed the ICMJE uniform disclosure form (available at http://dx. doi. org/10.21037/jtd-20-1610). The authors have no conflicts of interest to declare.

Ethical Statement: The authors are accountable for all aspects of the work in ensuring that questions related to the accuracy or integrity of any part of the work are appropriately investigated and resolved. This study was conducted in accordance with the Declaration of Helsinki (as revised in 2013) and approved by the institutional review board of Aichi Cancer Center, Aichi Prefecture, Japan (2019-1-544). Informed consent was waived due to the retrospective nature of this study.

Open Access Statement: This is an Open Access article distributed in accordance with the Creative Commons Attribution-NonCommercial-NoDerivs 4.0 International License (CC BY-NC-ND 4.0), which permits the noncommercial replication and distribution of the article with the strict proviso that no changes or edits are made and the original work is properly cited (including links to both the formal publication through the relevant DOI and the license). See: https://creativecommons.org/licenses/by-nc-nd/4.0/.

\section{References}

1. Chansky K, Detterbeck FC, Nicholson AG, et al. The IASLC lung cancer staging project: external validation of the revision of the TNM stage groupings in the eighth edition of the TNM classification of lung cancer. J Thorac Oncol 2017;12:1109-21.

2. Okada M, Nishio W, Sakamoto T, et al. Discrepancy of computed tomographic image between lung and 
mediastinal windows as a prognostic implication in small lung adenocarcinoma. Ann Thorac Surg 2003;76:1828-32.

3. Suzuki K, Koike T, Asakawa T, et al. Japan Lung Cancer Surgical Study Group (JCOG LCSSG). A prospective radiological study of thin-section computed tomography to predict pathological noninvasiveness in peripheral clinical IA lung cancer (Japan Clinical Oncology Group 0201). J Thorac Oncol 2011;6:751-6.

4. Sakao Y, Nakazono T, Sakuragi T, et al. Predictive factors for survival in surgically resected clinical IA peripheral adenocarcinoma of the lung. Ann Thorac Surg 2004;77:1157-61.

5. Sakao Y, Nakazono T, Tomimitsu S, et al. Lung adenocarcinoma can be subtyped according to tumor dimension by computed tomography mediastinalwindow setting. Additional size criteria for clinical T1 adenocarcinoma. Eur J Cardiothorac Surg 2004;26:1211-15.

6. Sakakura N, Inaba Y, Yatabe Y, et al. Estimation of the pathological invasive size of pulmonary adenocarcinoma using high-resolution computed tomography of the chest: A consideration based on lung and mediastinal window settings. Lung Cancer 2016;95:51-6.

7. Saito H, Kameda Y, Masui K, et al. Correlations between thin section CT findings, histopathological and clinical findings of small pulmonary adenocarcinomas. Lung Cancer 2011;71:137-43.

8. Sakao Y, Kuroda H, Mun M, et al. Prognostic significance of tumor size of small lung adenocarcinomas evaluated with mediastinal window settings on computed tomography. PLoS One 2014;9:e110305.

9. Kim H, Goo JM, Kim YT, et al. Consolidation-to-tumor ratio and tumor disappearance ratio are not independent prognostic factors for the patients with resected lung adenocarcinomas. Lung Cancer 2019;137:123-8.

10. Beasley MB, Brambilla E, Travis WD. The 2004 World

Cite this article as: Kuroda H, Nakada T, Oya Y, Takahashi Y, Matsusita H, Sakakura N. Clinical adjustability of radiological tools in patients with surgically resected cT1N0-staged nonsmall-cell lung cancer from the long-term survival evaluation. J Thorac Dis 2020;12(11):6655-6662. doi: 10.21037/jtd-20-1610
Health Organization classification of lung tumors. Semin Roentgenol 2005;40:90-7.

11. Sakakura N, Mizuno T, Kuroda H, et al. The eighth TNM classification system for lung cancer: A consideration based on the degree of pleural invasion and involved neighboring structures. Lung Cancer 2018;118:134-8.

12. Goldstraw P, Chansky K, Crowley J, et al. The IASLC lung cancer staging project: proposals for revision of the TNM stage groupings in the forthcoming (eighth) edition of the TNM classification for lung cancer. J Thorac Oncol 2016;11:39-51.

13. Rami-Porta R, Bolejack V, Crowley J, et al. The IASLC lung cancer staging project: proposals for the revisions of the $\mathrm{T}$ descriptors in the forthcoming eighth edition of the TNM classification for lung cancer. J Thorac Oncol 2015;10:990-1003.

14. Ye T, Deng L, Xiang J, et al. Predictors of Pathologic Tumor Invasion and Prognosis for Ground Glass Opacity Featured Lung Adenocarcinoma. Ann Thorac Surg 2018;106:1682-90.

15. Hattori A, Matsunaga T, Takamochi K, et al. Neither maximum tumor size nor solid component size is prognostic in part-solid lung cancer: impact of tumor size should be applied exclusively to solid lung cancer. Ann Thorac Surg 2016;102:407-15.

16. Ye T, Deng L, Wang S, et al. Lung Adenocarcinomas Manifesting as Radiological Part-Solid Nodules Define a Special Clinical Subtype. J Thorac Oncol 2019;14:617-27.

17. Kuroda H, Mori S, Tanaka H, et al. Prognostic significance of combined radiologic imaging modalities for prognosis of clinical IA adenocarcinomas. Oncotarget 2017;9:10745-53.

18. Peng M, Li Z, Hu H, et al. Pulmonary ground-glass nodules diagnosis: mean change rate of peak CT number as a discriminative factor of pathology during a follow-up. Br J Radiol 2016;89:20150556. 